\title{
Involvement of neuronal pathways in the protective effects of hindlimb perconditioning during renal ischemia
}

\author{
ZAHRA SEDAGHAT ${ }^{1}$, MEHRI KADKHODAEE $^{2}$, BEHJAT SEIFI $^{2}$, PARISA AHGHARI $^{3}$, \\ KHALIL POURKHALILI $^{1}$, ZAHRA AKBARI ${ }^{1}$ and MEHDI SADEGHI ${ }^{1}$ \\ ${ }^{1}$ Department of Physiology, Bushehr University of Medical Sciences, School of Medicine, \\ Bushehr 7514633341; ${ }^{2}$ Department of Physiology, Tehran University of Medical Sciences, \\ School of Medicine; ${ }^{3}$ Department of Physiology, Tehran University of Medical Sciences \\ (International Branch), School of Medicine, Tehran 1417613151, Iran
}

Received October 2, 2015; Accepted November 10, 2016

DOI: $10.3892 / \mathrm{etm} .2017 .4175$

\begin{abstract}
Remote ischemic perconditioning (RPEC) is a therapeutic intervention that has been demonstrated to reduce renal ischemia/reperfusion ( $\mathrm{I} / \mathrm{R})$ injury. However, the underlying renal protective mechanism remains unclear. The present study hypothesized that RPEC may utilize neural pathways to transfer the protective signal from the perconditioned hindlimb to the kidney. Following a right nephrectomy, rats were randomly allocated into five groups $(n=6)$. The sham group underwent the surgical protocol only. In all other groups, the left renal pedicle was clamped for $45 \mathrm{~min}$ and reperfused for $24 \mathrm{~h}$. The I/R control group then underwent $45 \mathrm{~min}$ ischemia and $24 \mathrm{~h}$ reperfusion $(\mathrm{I} / \mathrm{R})$ with no more intervention but the I/R-NR control group underwent the ischemia and reperfusion followed by left femoral nerve (FN) and sciatic nerve (SN) resection. The RPEC group underwent ischemia and reperfusion followed by four cycles of $5 \mathrm{~min}$ occlusions of the left femoral artery and 5 min reperfusion. Finally, the RPEC-NR group underwent ischemia and reperfusion followed by left $\mathrm{FN}$ and SN resection. Following $24 \mathrm{~h}$, renal functional indices, plasma blood urea nitrogen (BUN) and creatinine (Cr) levels, urinary $N$-acetyl- $\beta$-glucosaminidase (NAG) release and histopathological changes were assessed. Compared with the sham group, ischemia and reperfusion in the sham and I/R control groups resulted in renal dysfunction, indicated by significantly increased levels of BUN and Cr. This was accompanied by increased urinary NAG activity and morphological damage observed in control groups. In the RPEC group, renal histology and function were significantly improved compared with the
\end{abstract}

Correspondence to: Dr Zahra Sedaghat, Department of Physiology, Bushehr University of Medical Sciences, School of Medicine, 101 Moallem Street, Bushehr 7514633341, Iran

E-mail: z.sedaghat@bpums.ac.ir

Key words: perconditioning, kidney, ischemia/reperfusion, neuronal pathways, remote organ control groups. However, FN and SN resection eliminated the protection of the kidney, which was induced by RPEC. In conclusion, remote hindlimb ischemic perconditioning reduced renal $\mathrm{I} / \mathrm{R}$ injury in the rat kidney in a manner that potentially involves a neural pathway.

\section{Introduction}

Renal ischemia/reperfusion (I/R) injury occurs following reperfusion of the ischemic kidney in a number of clinical scenarios, including kidney transplantation (1). Renal I/R injury presents as acute renal functional impairment and acute tubular necrosis. These events substantially contribute to renal-associated morbidity and mortality, following acute kidney injury (2).

A number of studies have assessed the innate protective mechanisms that attenuate the deleterious effects of $I / R$ injury (3). The phenomenon of ischemic preconditioning (IPC) has been implicated to protect the kidney from ischemic injury, following studies that applied a short cycle of $I / R$ to the kidney prior to a severe ischemic event (4). However, preconditioning has clinical limitations in the case of acute unpredicted ischemic scenarios (5). Remote perconditioning (RPEC) is defined as repeated periods of alternating ischemia and reperfusion in the limbs, applied at the beginning of renal ischemia (3). RPEC has previously been demonstrated to be a potentially useful method in the manipulation of innate renoprotection (6). The concept of RPEC is a clinically amenable strategy, in which the ischemic perconditioning cycles of $I / R$ are applied to an organ other than the kidney and therefore, may be beneficial in unpredicted acute ischemic conditions. However, the mechanism of protection exerted by RPEC has not been fully established. Furthermore, the mechanism through which the renoprotective signal is conveyed, from the perconditioned limb to the kidney, remains unclear and has been ascribed to a neural or humoral pathway (3).

The activation of the complicated neural mechanisms may be caused by the release of different mediators such as adenosine (7) and bradykinin (8) from the ischemic conditioned remote organ during the cyclic sublethal I/R and stimulation of local neural components. It has been suggested 
that these mediators, as possible triggers, are able to induce capsaicin-sensitive sensory neurons to release calcitonin gene related peptides. Thus, activating a sub-cellular cascade of kinases as a protective mechanism (9). Previous studies have indicated that manipulation of hindlimb innervations prior to induction of ischemic perconditioning treatment exerts an effect on perconditioning-induced renoprotection $(10,11)$.

Our previous study was the first to report the protective effects of RPEC on the I/R injury of the kidney (12). The current study aimed to evaluate the effects of hindlimb ischemic perconditioning and thus, approve or disapprove the involvement of intact remote organ innervation in transduction of protective signals.

\section{Materials and methods}

Animals. A total of 30 Male Sprague-Dawley rats (weight, 220-270 g; age, 7-8 weeks) were housed under standard conditions (12-h light/dark cycle; $20-22^{\circ} \mathrm{C}$ ) and had ad libitum access to water and standard pellet diet. Prior to surgery, anesthesia was administered by an intraperitoneal injection of $60 \mathrm{mg} / \mathrm{kg}$ sodium pentobarbital (Sigma-Aldrich; Merck KGaA, Darmstadt, Germany). Systolic blood pressure and heart rate were recorded during the surgery using a tail cuff linked to a pulse transducer (MLT125/; ADInstruments, Inc., Sydney, Australia), connected to a PowerLab/4SP data acquisition system (software version 5; ADInstruments, Inc.). Animals that presented with hypotension $(<60 \mathrm{mmHg})$ were excluded from the subsequent experiments; however, in the present study none of the animals showed hypotension. All procedures were approved by the Animal Ethics Committee of Bushehr University of Medical Sciences (Tehran, Iran).

Surgical procedure. Prior to renal ischemia induction, a nerve resection was completed in three groups of this study which is specified at the following section. The left femoral artery, femoral and sciatic nerves were separated from surrounding connective tissue. The femoral nerve was then separated from the femoral artery and vein at the proximal area near the groin. Renal ischemia was performed by occlusion of the renal pedicle with a bulldog clamp. RPEC protocol was induced by applying four cycles of $5 \mathrm{~min}$ ischemia and $5 \mathrm{~min}$ reperfusion of the left femoral artery with an atraumatic microvessel clamp, just at the beginning of the renal ischemia. Following the surgical procedure, animals were kept in metabolic cages and urine samples collection performed over a period of $24 \mathrm{~h}$. At the end of the reperfusion time, rats were anesthetized with sodium pentobarbital $(60 \mathrm{mg} / \mathrm{kg})$ and blood samples were obtained using a 5-ml syringe. Following sacrifice, the chests of the rats were opened and their kidneys were resected and fixed in $10 \%$ formalin for subsequent histological study.

Experimental groups. All animals underwent a right nephrectomy before the induction of ischemia, which was accomplished through a small flank incision. Rats were randomly assigned to one of five groups ( $n=6$ per group): i) Sham-operated group: Femoral and sciatic nerve resection plus sham surgery; ii) Control group (I/R): 45 min of renal ischemia by occlusion of the left renal pedicle; iii) Control group and nerve resection (I/R-NR): $45 \mathrm{~min}$ ischemia with femoral and sciatic nerve resection; iv) RPEC: 4 cycles of 5 min left femoral artery occlusion and reperfusion just prior to renal ischemia; or v) Nerve resection group (RPEC-NR): RPEC protocol plus femoral and sciatic nerve resection prior to induction of renal ischemia.

Renal functional assessment. At the end of the reperfusion period, blood samples were drawn via the inferior vena cava using a syringe. The samples were centrifuged at room temperature $(1,500 \mathrm{x} \mathrm{g}$ for $10 \mathrm{~min})$ to separate the plasma. In order to assess the glomerular functions, the concentration of blood urea nitrogen (BUN) and creatinine $(\mathrm{Cr})$ were measured by colorimetric methods (Hitachi 704 autoanalyser; Hitachi, Ltd., Tokyo, Japan).

Urinary $N$-acetyl- $\beta$-D-glucosaminidase (NAG) analysis. NAG is a lysosomal glycosidase found in high concentrations in renal proximal tubules. Activity of urinary NAG, a specific indicator of early tubular injury, was measured according to the method described by Horak et al (13). The total volume of produced urine during the $24 \mathrm{~h}$ reperfusion time was noted, and samples were kept at $-20^{\circ} \mathrm{C}$ until use. The assessment of urinary NAG activity was based on the enzymatic hydrolysis of p-nitrophenyl-n-acetyl-glucosaminide at $\mathrm{pH} 4.4$ and the subsequent production of p-nitrophenol at $405 \mathrm{~nm}$ by using a spectrophotometer (Spekol 1300; Analytik Jena AG, Jena, Germany).

Histopathological analysis. Samples from the kidney of all rats were harvested following $24 \mathrm{~h}$ reperfusion and fixed in $10 \%$ formalin. The fixed tissue specimens were dehydrate, embedded in paraffin, sectioned at $4-\mu \mathrm{m}$, and stained with hematoxylin and eosin for histological examination under a light microscope. The sections were assessed for the presence of tubular necrosis, tubular dilatations and loss of brush borders, as well as the formation of casts and luminal debris at x400 magnification.

Statistical analysis. Statistical analyses were performed using GraphPad Prism Version 5 software (GraphPad Software, Inc., La Jolla, CA, USA). The results are presented as mean \pm standard error of the mean. The differences among groups were determined by one-way analysis of variance followed by Tukey's multiple comparison post hoc test. $\mathrm{P}<0.05$ was considered to represent a statistically significant difference.

\section{Results}

Renal function and levels of plasma BUN and $\mathrm{Cr}$. Following $24 \mathrm{~h}$ reperfusion, the levels of BUN and $\mathrm{Cr}$ in the plasma were $21.7 \pm 1.1$ and $0.65 \pm 0.14 \mathrm{mg} / \mathrm{dl}$, respectively in the sham group. Compared with the sham group, the levels of BUN and $\mathrm{Cr}$ in the I/R and IR-NR groups were significantly increased (5-fold) following $45 \mathrm{~min}$ of ischemia and $24 \mathrm{~h}$ of reperfusion (BUN, $117.0 \pm 5.3$ and $111.7 \pm 8.2 \mathrm{mg} / \mathrm{dl} ; \mathrm{Cr}, 4.1 \pm 0.5$ and $3.9 \pm 0.7 \mathrm{mg} / \mathrm{dl}$, respectively; $\mathrm{P}<0.05)$. In the RPEC group, the level of BUN $(47.6 \pm 6.2 \mathrm{mg} / \mathrm{dl})$ and plasma $\mathrm{Cr}(1.2 \pm 0.3 \mathrm{mg} / \mathrm{dl})$ were significantly lower compared with those in I/R and I/R-NR groups $(\mathrm{P}<0.05)$. However, the remote perconditioning-induced 
A

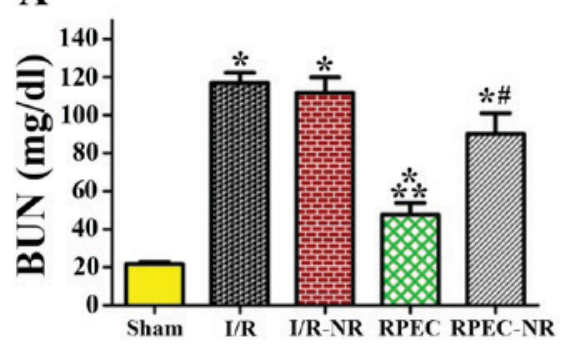

B

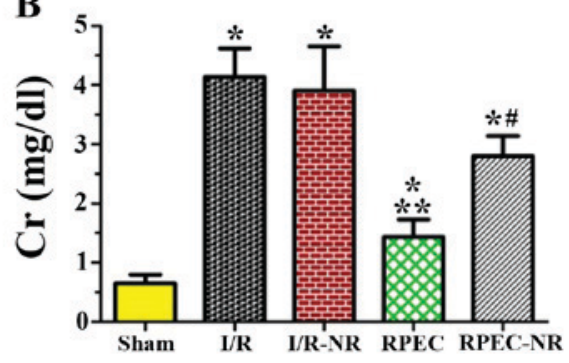

Figure 1. Serum levels of BUN and Cr among the different groups. The levels of (A) BUN and (B) Cr in each group. Each column and bar represents the mean \pm standard error of the mean ( $\mathrm{n}=6$ per group). $\mathrm{P}<0.05$ vs. sham group; ${ }^{* *} \mathrm{P}<0.05 \mathrm{vs}$. I/R group; ${ }^{\#} \mathrm{P}<0.05 \mathrm{vs}$. RPEC group. I/R, ischemia/reperfusion group; I/R-NR, ischemia/reperfusion plus femoral and sciatic nerve resection group; RPEC, remote perconditioning group; RPEC-NR, remote preconditioning plus femoral and sciatic nerves resection group; BUN, blood urea nitrogen; $\mathrm{Cr}$, creatinine.

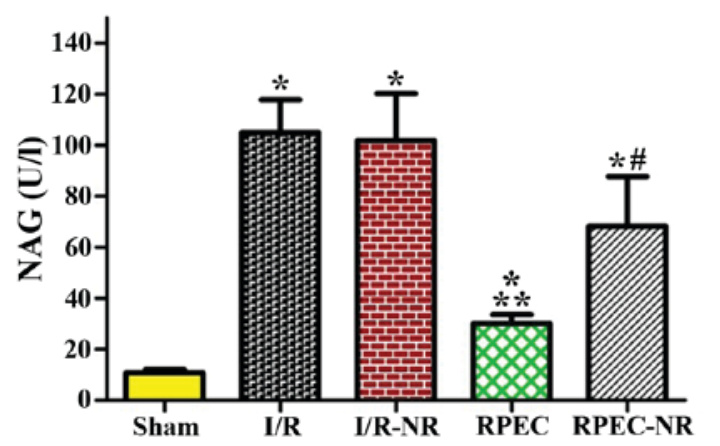

Figure 2. Levels of blood urinary NAG among the different groups. Each column and bar represents the mean \pm standard error of the mean $(n=6$ per group). ${ }^{*} \mathrm{P}<0.05$ vs. sham group; ${ }^{* *} \mathrm{P}<0.05$ vs. I/R group; ${ }^{*} \mathrm{P}<0.05$ vs. RPEC group. I/R, ischemia/reperfusion group; I/R-NR, ischemia/reperfusion plus femoral and sciatic nerves resection group; RPEC, remote perconditioning group; RPEC-NR, remote preconditioning plus femoral and sciatic nerves resection group; NAG, $\mathrm{N}$-acetyl- $\beta$-D-glucosaminidase.

improvement was significantly inhibited by the nerve resection in RPEC-NR group (BUN, 90.2 $\pm 10.8 \mathrm{mg} / \mathrm{dl} ; \mathrm{Cr}$, $2.8 \pm 0.3 \mathrm{mg} / \mathrm{dl}$; Fig. 1).

Renal injury and urinary NAG activity. The urinary NAG activity in the sham group was $10.8 \pm 1.4 \mathrm{U} / 1$. Compared with the sham group, the urinary NAG activity in the I/R and IR-NR groups was significantly increased ( 10-fold) following $45 \mathrm{~min}$ ischemia and $24 \mathrm{~h}$ of reperfusion $(105.1 \pm 12.8$ and $101.8 \pm 18.5 \mathrm{U} / 1$ respectively; $\mathrm{P}<0.05)$. In the RPEC group, the level of urinary NAG $(30.1 \pm 3.6 \mathrm{mg} / \mathrm{dl})$ was significantly lower compared with those in $\mathrm{I} / \mathrm{R}$ and $\mathrm{I} / \mathrm{R}-\mathrm{NR}(\mathrm{P}<0.05)$. This remote perconditioning-induced improvement was significantly inhibited by nerve resection in the RPEC-NR group (68.3 $\pm 19.5 \mathrm{U} / 1$; Fig. 2).

Histological changes. Notable histopathological changes were not observed in the kidney sections of the sham group. However, renal IR resulted in considerable changes in the renal tissues. The alteration observed in the control groups (I/R and I/R-NR groups) included severe destruction of renal tubules caused by cellular necrosis, specifically in the proximal tubules, formation of cast and obstruction of the more distal tubules. The brush borders were lost and tubular patency was attenuated. Perconditioning reduced the extent of structural changes in the kidney, compared with the I/R and I/R-N groups. Noticeable
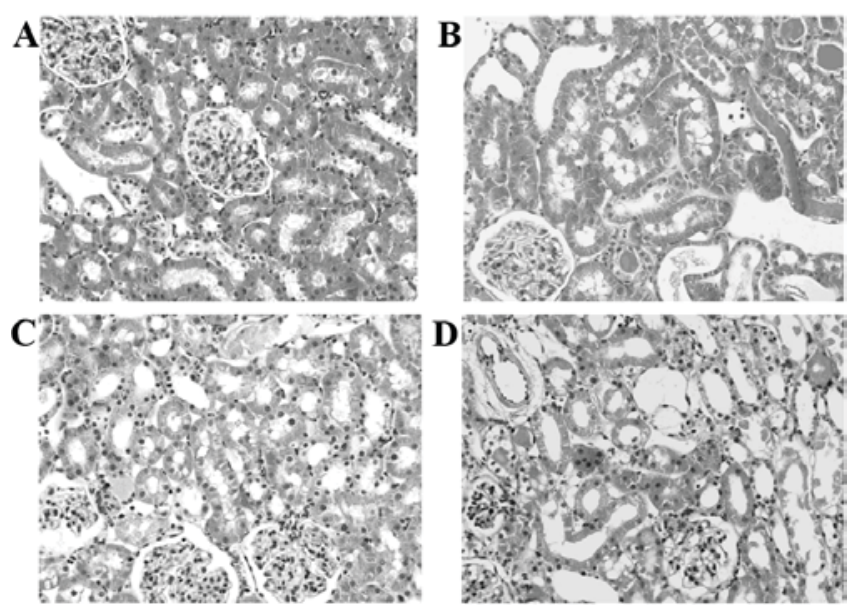

Figure 3. Renal histological changes in the kidney. (A) Sham group (B) ischemia/reperfusion group, (C) remote perconditioning and (D) remote preconditioning plus femoral and sciatic nerves resection. The histological features of I/R-NR group was not different from the I/R group (not included in this figure). Hematoxylin and eosin stained sections were evaluated by light microscopy at x400 magnification.

cellular necrosis was not detected in the RPEC-treated group and there was less cast formation in the tubules. However, NR prior to perconditioning treatment (RPEC-NR group) was observed to cause more histological damage compared with that observed in the animals of RPEC group (Fig. 3).

\section{Discussion}

The current study demonstrates the renoprotective effects of RPEC, achieved via short-cycle I/R of the hindlimb in a rat model of renal ischemia and reperfusion-induced acute kidney injury. To the best of our knowledge, the present study is the first to propose the potential contribution of neural pathways in the transduction of reno-protective signals elicited by remote perconditioning.

The mechanism underlying remote perconditioning therapy remains unknown, with a number of previous studies collecting data from cardiological and hepatic studies that used different remote ischemic conditioning models $(14,15)$. There is limited data available regarding the ischemic features of the kidney and to the best of our knowledge, no data has been reported assessing the role of neural pathways leading to the renoprotective outcome of RPEC. In a previous study, 
Lim et al (10) reported that the cardioprotection caused by hindlimb preconditioning is completely eradicated following cross-section of the femoral nerve and sciatic nerve. Based on these results and previous data (6), the present study aimed to assess the effects of the limb nerve cross-section on the efficiency of remote perconditioning to mitigate renal I/R injury.

Ischemia and reperfusion of the kidney result in both glomerular and tubular malfunction (16). In the present study, the significant increases in the plasma levels of creatinine and BUN, subsequent to I/R, indicate an impairment of glomerular function. However, I/R also resulted in significant increases in urinary NAG activities, which may be regarded as a marker for tubular injury (17). Perconditioning was able to significantly reduce renal functional impairment, this is indicated by a decreased level of BUN and $\mathrm{Cr}$ in the plasma as well as reduced urinary NAG activity, the activity of which was abrogated by nerve resection.

In accordance with results from the present study, the extent of renal histological damages was prominent and extended in the I/R damaged groups (IR and IR-N) at the end of reperfusion time. Perconditioning induction diminished the extent of these injured tubules, although such protective effects were not observed in the RPEC-NR group where cross-section of the nerve prior to the ischemic conditioning treatment provoked renal morphological changes. The notable infarct size reducing effects of remote perconditioning were also reported by Lu et al (18) and Czigány et al (11) in different experimental methods of myocardial infarction and liver $\mathrm{I} / \mathrm{R}$.

It is suggested that the mechanisms involved in local and remote preconditioning and postconditioning models may also have roles in the RPEC treatment (3). These mechanisms include the release of a number of protective autocoids such as adenosine (19) and nitric oxide (20), the activation of the innate immune system (21), activation of the reperfusion injury salvage kinase pathway (22) and the inhibition of the mitochondrial permeability transition pore (23). Moreover, we have recently reported the renoprotective effect of remote perconditioning by modulation of inflammatory cytokines in the kidney (24) and attenuated oxidative damages in more distantly located organs, such as the liver (25). Supporting the involvement of a neurogenic pathway, the protective effects of different ischemic conditioning strategies such as remote IPC may be suppressed by ganglionic blockers (26). In addition, remote IPC using short-time limb ischemia and reperfusion may be blocked by prior cross-section of the femoral nerves (10). Previous evidence indicates that autocoids such as adenosine or bradykinin may activate a local neural path within the ischemic conditioned limb (8). A study by Liem et al (27) proposed that the release of adenosine by the remote perconditioned small intestine triggers local afferent nerves and thereby protects the heart from consequent $\mathrm{I} / \mathrm{R}$ damage via triggering the adenosine receptors in the myocardium. These data are in line with the results of the present study, indicating a possible role of neuronal elements in the conduction of protective signals from the remote conditioned site to the ischemic organ.

In conclusion, the results of the present study have indicated that the renoprotective effect of RPEC may be mitigated by nerve resection of the remote ischemic conditioned organ. Therefore, the current study indicates that the protective signals elicited by remote perconditioning, to a significant extent, are directed to the target ischemic organ by certain neural factors. In the present study, the molecular and cellular details of the neural pathway behind the protective effects of RPEC were not assessed, which is a limitation. Accordingly, further investigation should be conducted to reveal the exact molecular and cellular mechanisms and neural components behind the protective effect of remote ischemic perconditioning treatment.

\section{Acknowledgements}

This study was supported by a grant from Bushehr University of Medical Sciences (grant no. IR.BPUMS.1395.157) to Dr Zahra Sedaghat.

\section{References}

1. Cooper JE and Wiseman AC: Acute kidney injury in kidney transplantation. Curr Opin Nephrol Hypertens 22: 698-703, 2013.

2. Glodowski SD and Wagener G: New insights into the mechanisms of acute kidney injury in the intensive care unit. J Clin Anesth 27: 175-180, 2014.

3. Hausenloy DJ and Yellon DM: Ischaemic conditioning and reperfusion injury. Nat Rev Cardiol 13: 193-209, 2016.

4. Hu J, Liu S, Jia P, Xu X, Song N, Zhang T, Chen R and Ding X: Protection of remote ischemic preconditioning against acute kidney injury: A systematic review and meta-analysis. Crit Care 20: 111, 2016.

5. McCafferty K, Byrne C and Yaqoob MM: Ischaemic conditioning strategies for the nephrologist: A promise lost in translation? Nephrol Dial Transplant 29: 1827-1840, 2014

6. Sedaghat Z, Kadkhodaee M, Seifi B, Salehi E, Najafi A and Dargahi L: Remote perconditioning reduces oxidative stress, downregulates cyclo-oxygenase- 2 expression and attenuates ischaemia-reperfusion-induced acute kidney injury. Clin Exp Pharmacol Physiol 40: 97-103, 2013.

7. Liem DA, Verdouw PD, Ploeg H, Kazim S and Duncker DJ: Sites of action of adenosine in interorgan preconditioning of the heart. Am J Physiol Heart Circ Physiol 283: H29-H37, 2002.

8. Schoemaker RG and van Heijningen CL: Bradykinin mediates cardiac preconditioning at a distance. Am J Physiol Heart Circ Physiol 278: H1571-H1576, 2000.

9. Wolfrum S, Nienstedt J, Heidbreder M, Schneider K, Dominiak $\mathrm{P}$ and Dendorfer A: Calcitonin gene related peptide mediates cardioprotection by remote preconditioning. Regul Pept 127: 217-224, 2005.

10. Lim SY, Yellon DM and Hausenloy DJ: The neural and humoral pathways in remote limb ischemic preconditioning. Basic Res Cardiol 105: 651-655, 2010.

11. Czigány Z, Turóczi Z, Kleiner D, Lotz G, Homeyer A, Harsányi L and Szijártó A: Neural elements behind the hepatoprotection of remote perconditioning. J Surg Res 193: 642-651, 2015 .

12. Kadkhodaee M, Seifi B, Najafi A and Sedaghat Z: First report of the protective effects of remote per- and postconditioning on ischemia/reperfusion-induced renal injury. Transplantation 92: e55, 2011.

13. Horak E,Hopfer SM and Sunderman FW Jr: Spectrophotometric assay for urinary $\mathrm{N}$-acetyl-beta-D-glucosaminidase activity. Clin Chem 27: 1180-1185, 1981.

14. Zaman J, Jeddi S and Ghasemi A: The effects of ischemic postconditioning on myocardial function and nitric oxide metabolites following ischemia-reperfusion in hyperthyroid rats. Korean J Physiol Pharmacol 18: 481-487, 2014.

15. Chai Q, Liu J and $\mathrm{Hu}$ Y: Cardioprotective effect of remote preconditioning of trauma and remote ischemia preconditioning in a rat model of myocardial ischemia/reperfusion injury. Exp Ther Med 9: 1745-1750, 2015.

16. Zuk A and Bonventre JV: Acute kidney injury. Annu Rev Med 67: 293-307, 2016.

17. Schmid M, Dalela D, Tahbaz R, Langetepe J, Randazzo M, Dahlem R, Fisch M, Trinh QD and Chun FK: Novel biomarkers of acute kidney injury: Evaluation and evidence in urologic surgery. World J Nephrol 4: 160-168, 2015. 
18. Lu Y, Hu J and Dong C: Morphine may enhance the cardioprotection induced by remote ischemic perconditioning. Int $\mathrm{J}$ Cardiol 187: 443-444, 2015.

19. Yun J, Li J and Zuo Z: Transferred inter-cell ischemic preconditioning-induced neuroprotection may be mediated by adenosine A1 receptors. Brain Res Bull 103: 66-71, 2014.

20. Rassaf T, Totzeck M, Hendgen-Cotta UB, Shiva S, Heusch G and Kelm M: Circulating nitrite contributes to cardioprotection by remote ischemic preconditioning. Circ Res 114: 1601-1610, 2014.

21. Shimizu M, Saxena P, Konstantinov IE, Cherepanov V, Cheung MM, Wearden P, Zhangdong H, Schmidt M, Downey GP and Redington AN: Remote ischemic preconditioning decreases adhesion and selectively modifies functional responses of human neutrophils. J Surg Res 158: 155-161, 2010.

22. Skyschally A, Gent S, Amanakis G, Schulte C, Kleinbongard P and Heusch G: Across-Species transfer of protection by remote ischemic preconditioning with species-specific myocardial signal transduction by reperfusion injury salvage kinase and survival activating factor enhancement pathways. Circ Res 117: 279-288, 2015.
23. Turrell HE, Thaitirarot $\mathrm{C}$, Crumbie $\mathrm{H}$ and Rodrigo G: Remote ischemic preconditioning of cardiomyocytes inhibits the mitochondrial permeability transition pore independently of reduced calcium-loading or sarcKATP channel activation. Physiol Rep 2: e12231, 2014

24. Sedaghat Z, Kadkhodaee M, Seifi B and Salehi E: Hind limb perconditioning renoprotection by modulation of inflammatory cytokines after renal ischemia/reperfusion. Ren Fail 38: 655-662, 2016.

25. Sedaghat Z, Kadkhodaee M, Seifi B and Ahghari P: Hepatoprotective effects of remote perconditioning during renal ischemia. Bratisl Lek Listy 115: 675-679, 2014.

26. Loukogeorgakis SP, Panagiotidou AT, Broadhead MW, Donald A, Deanfield JE and MacAllister RJ: Remote ischemic preconditioning provides early and late protection against endothelial ischemia-reperfusion injury in humans: Role of the autonomic nervous system. J Am Coll Cardiol 46: 450-456, 2005.

27. Liem DA, Verdouw PD, Ploeg H, Kazim S and Duncker DJ: Sites of action of adenosine in interorgan preconditioning of the heart. Am J Physiol Heart Circ Physiol 283: 29-37, 2002. 